\title{
A CLASS OF NONSYMMETRIC HARMONIC RIEMANNIAN SPACES
}

\author{
EWA DAMEK AND FULVIO RICCI
}

\begin{abstract}
Certain solvable extensions of $H$-type groups provide noncompact counterexamples to a conjecture of Lichnerowicz, which asserted that "harmonic" Riemannian spaces must be rank 1 symmetric spaces.
\end{abstract}

A Riemannian space $M$ with Laplace-Beltrami operator $\Delta$ is called harmonic if, given any function $f(x)$ on $M$ depending only on the distance $d\left(x, x_{0}\right)$ from a given point $x_{0}$, then also $\Delta f(x)$ depends only on $d\left(x, x_{0}\right)$.

Equivalently, $M$ is harmonic if for every $p \in M$ the density function $\omega_{x_{0}}(x)$ expressed in terms of the normal coordinates around the point $x_{0}$ is a function of $d\left(x, x_{0}\right)$ (see $\left.[1,11]\right)$.

In 1944 Lichnerowicz [10] proved that in dimensions not greater than 4 the harmonic spaces coincide with the rank-one symmetric spaces. He also raised the question of determining whether the same is true in higher dimensions.

Among the most recent progress made on the so-called Lichnerowicz conjecture, Szabó [11] proved it to hold true in arbitrary dimension for compact manifolds with finite fundamental group.

In this announcement we present a counterexample that arises in the noncompact case. It proves the Lichnerowicz conjecture not to be true in general for infinitely many dimensions, the smallest of them being 7 .

This example is based on the notion of H-type group due to Kaplan [8], and on the geometric properties of their one-dimensional extensions $S$ introduced by Damek [5] and studied also in [2, 3, 4, 6].

The rank-one symmetric spaces of the noncompact type different from the hyperbolic spaces are special examples of such groups $S$. Even though symmetry is a main geometric difference that distinguishes some "good" $S$ from other "bad" $S$, it turns out that a large part of the harmonic analysis on these groups can be worked out regardless of this distinction.

A detailed account of this is given in our forthcoming paper [7].

We thank J. Faraut and A. Korányi for calling our attention to the Lichnerowicz conjecture.

\section{The EXTENSION $S$ OF AN H-TYPE GROUP}

An H-type (or Heisenberg-type) algebra is a two-step nilpotent Lie algebra $\mathfrak{n}$ with an inner product $\langle$,$\rangle such that if \mathfrak{z}$ is the center of $\mathfrak{n}$ and $\mathfrak{v}=\mathfrak{z}^{\perp}$, then the map $J_{Z}: \mathfrak{v} \rightarrow \mathfrak{v}$ given by

$$
\left\langle J_{Z} X, Y\right\rangle=\langle[X, Y] . Z\rangle
$$

for $X, Y \in \mathfrak{v}$ and $Z \in \mathfrak{z}$, satisfies the identity $J_{Z}^{2}=-|Z|^{2} I$ for every $Z \in \mathfrak{z}$.

Received by the editors July 11, 1991.

1991 Mathematics Subject Classification. Primary 53C25, 53C30, 43A85, 22E25, 22 E30. 
An H-type group is a connected and simply connected Lie group $N$ whose Lie algebra is $\mathrm{H}$-type.

Let $\mathfrak{s}=\mathfrak{v} \oplus \mathfrak{z} \oplus \mathbb{R} T$ be the extension of $\mathfrak{n}$ obtained by adding the rule

$$
[T, X+Z]=\frac{1}{2} X+Z
$$

for $X \in \mathfrak{v}$ and $Z \in \mathfrak{z}$. Let $S=N A$ be the corresponding connected and simply connected group extension of $N$, where $A=\exp _{S}(\mathbb{R} T)$. We parametrize the elements of $S$ in terms of triples $(X, Z, a) \in \mathfrak{v} \oplus \mathfrak{z} \oplus \mathbb{R}^{+}$by setting

$$
(X, Z, a)=\exp _{S}(X+Z) \exp _{S}(\log a T) \text {. }
$$

The product on $S$ is then

$$
(X, Z, a)\left(X^{\prime}, Z^{\prime}, a^{\prime}\right)=\left(X+a^{1 / 2} X^{\prime}, Z+a Z^{\prime}+\frac{1}{2} a^{1 / 2}\left[X, X^{\prime}\right], a a^{\prime}\right) \text {. }
$$

We introduce the inner product

$$
\left\langle X+Z+t T \mid X^{\prime}+Z^{\prime}+t^{\prime} T\right\rangle=\left\langle X \mid X^{\prime}\right\rangle+\left\langle Z \mid Z^{\prime}\right\rangle+t t^{\prime}
$$

on $\mathfrak{s}$ and endow $S$ with the induced left-invariant Riemannian metric.

Proposition 1 [4, 5, 9]. Let $M=G / K$ be a rank-one symmetric space of the noncompact type different from $S O_{e}(n, 1) / S O(n)$, and let $G=N A K$ be the Iwasawa decomposition of $G$. Then $N$ is an H-type group and the map $s \mapsto s K$ is an isometry from $S=N A$ to $G / K$.

From the classification of symmetric spaces one knows that if the H-type group $N$ appears in the Iwasawa decomposition of a rank-one symmetric space, then the dimension of its center equals 1,3 , or 7 . On the other hand, there exist H-type groups with centers of arbitrary dimensions [8]. Direct proofs can be given $[4,5]$ to show that the space $S$ is symmetric if and only if $N$ is an "Iwasawa group."

We can then conclude that there are infinitely many $S$ that are not symmetric.

\section{THE VOLUME ELEMENT IN RADIAL NORMAL COORDINATES}

Given unit elements $X_{0} \in \mathfrak{v}$ and $Z_{0} \in \mathfrak{z}$, we denote by $S_{X_{0}, Z_{0}}$ the 4dimensional subgroup of $S$ generated by $X_{0}, Z_{0}, J_{Z_{0}} X_{0}$, and $T$. Clearly, $S_{X_{0}, Z_{0}}$ is also the extension of an H-type group, precisely of the 3-dimensional Heisenberg group.

Proposition 2 [3]. $S_{X_{0}, Z_{0}}$ is a totally geodesic submanifold of $S$, isometric to the Hermitian symmetric space $S U(2,1) / S(U(2) \times U(1))$.

This observation suggests two alternative realizations of $S[4,7]$ :

(1) the "Siegel domain" model:

$$
D=\left\{(X, Z, t) \in \mathfrak{v} \oplus \mathfrak{z} \oplus \mathbb{R}: t>\frac{1}{4}|X|^{2}\right\}
$$

with the metric transported from $S$ via the map $h(X, Z, a)=(X, Z$, $\left.a+\frac{1}{4}|X|^{2}\right)$;

(2) the "ball" model:

$$
B=\left\{(X, Z, t) \in \mathfrak{v} \oplus \mathfrak{z} \oplus \mathbb{R}:|X|^{2}+|Z|^{2}+t^{2}<1\right\}
$$

with the metric transported from $D$ via the inverse of the "Cayley transform" $C: B \rightarrow D$ given by

$$
C(X, Z, t)=\frac{1}{(1-t)^{2}+|Z|^{2}}\left(2\left(1-t+J_{Z}\right) X, 2 Z, 1-t^{2}-|Z|^{2}\right) .
$$


The map $C^{-1} \circ h: S \rightarrow B$ maps the identity element $e=(0,0,1)$ to the origin and its differential at $e$ equals $\frac{1}{2} I$.

Let $m=\operatorname{dim} \mathfrak{v}, k=\operatorname{dim} \mathfrak{z}$. The number $Q=\frac{m}{2}+k$ is called the homogeneous dimension of $N$. It is easily checked that

$$
d m_{L}=a^{-Q-1} d X d Z d a
$$

is a left Haar measure on $S$ and is also the Riemannian volume element.

Applying Proposition 2, using the properties of the Cayley transform in the symmetric Hermitian case, and explicitely computing the Jacobians of $h$ and $C$, we obtain the following result.

Theorem 1 [7]. (1) In the metric on $B$ transported from $S$ via $C^{-1} \circ h$, the geodesics through the origin are the diameters and the distance $\rho$ from a point $(X, Z, t) \in B$ to the origin is a function of $r=\left(|X|^{2}+|Z|^{2}+t^{2}\right)^{1 / 2}$, namely,

$$
\rho=\log \frac{1+r}{1-r} \text {. }
$$

(2) Introducing polar coordinates $(r, \omega)$ on $B$ and denoting by $d \sigma(\omega)$ the surface measure on $S^{m+k}$, the volume element on $B$ is given by

$$
2^{m+k+1}\left(1-r^{2}\right)^{-Q-1} r^{m+k} d r d \sigma(\omega)=2^{m+k}\left(\cosh \frac{\rho}{2}\right)^{k}\left(\sinh \frac{\rho}{2}\right)^{m+k} d \rho d \sigma(\omega) .
$$

The coordinates $(\rho, \omega)$ are the radial normal coordinates on $B$ around the origin. Then (2) proves that the volume density depends only on $\rho$. The same is true for normal coordinates around any other point, since $S$ and $B$ are isometric and $S$ is obviously homogeneous. We can then conclude that

Corollary. For every H-type group $N, S=N A$ is a harmonic space.

Consider now the Laplace-Beltrami operator $\Delta$ on $S$. Its extension to the domain

$$
\mathscr{D}(\Delta)=\left\{f \in L^{2}\left(S, d m_{L}\right): \Delta f \in L^{2}\left(S, d m_{L}\right)\right\}
$$

is selfadjoint and positive. From the spectral resolution

$$
\Delta=\int_{0}^{+\infty} \lambda d E_{\lambda}
$$

one constructs the heat semigroup

$$
T_{t}=e^{-t \Delta}=\int_{0}^{+\infty} e^{-t \lambda} d E_{\lambda}
$$

Since $\Delta$ is left-invariant,

$$
\left(T_{t} f\right)(x)=\left(f * p_{t}\right)(x)=\int_{S} f(y) p_{t}\left(y^{-1} x\right) d m_{L}(y)
$$

and $p(t, x)=p_{t}(x)$ is a smooth function on $\mathbb{R}^{+} \times S$. In [7] we also prove the following strong-harmonicity property of $S$. 
Theorem 2. The functions $p_{t}(x)$ depend only on the geodesic distance from $x$ to $e$.

\section{DiMENSIONS OF THE NONSYMMETRIC $S$}

A complete list of $\mathrm{H}$-type groups can be obtained from the classification of Clifford modules [8]. For small values of $k=\operatorname{dim} \mathfrak{z}$ we write the dimensions of the corresponding nonsymmetric $S$ :

$\begin{array}{ll}k & \operatorname{dim} S \\ 1 & - \\ 2 & 7+4 n \\ 3 & 12+4 n \\ 4 & 13+8 n \\ 5 & 14+8 n \\ 6 & 15+8 n \\ 7 & 24+8 n \\ 8 & 25+16 n\end{array}$

where $n=0,1, \ldots$.

\section{REFERENCES}

1. A. L. Besse, Manifolds all of whose geodesics are closed, Springer, Berlin, 1978.

2. J. Boggino, Generalized Heisenberg groups and solvmanifolds naturally associated, Rend. Sem. Mat. Univ. Polit. Torino 43 (1985), 529-547.

3. M. Cowling, A. H. Dooley, A. Korányi, and F. Ricci, H-type groups and Iwasawa decompositions, Adv. Math. 87 (1991), 1-41.

4. —_ H-type groups and Iwasawa decompositions, II (to appear).

5. E. Damek, Geometry of a semidirect extension of a Heisenberg type nilpotent group, Colloq. Math. 53 (1987), 255-268.

6. Curvature of a semidirect extension of a Heisenberg type nilpotent group, Colloq. Math. 53 (1987), 249-253.

7. E. Damek and F. Ricci, Harmonic analysis on solvable extensions of H-type groups, J. Geom. Anal. (to appear).

8. A. Kaplan, Fundamental solutions for a class of hypoelliptic PDE generated by compositions of quadratic forms, Trans. Amer. Math. Soc. 258 (1980), 147-153.

9. A. Korányi, Geometric properties of Heisenberg-type groups, Adv. Math. 56 (1985), 28-38.

10. A. Lichnerowicz, Sur les espaces Riemanniens complètement harmoniques, Bull. Soc. Math. France 72 (1944), 146-168.

11. Z. Szabó, The Lichnerowicz conjecture on harmonic manifolds, J. Differential Geom. 31 (1990), 1-28.

Instytut MatematyczNy Universytetu Wroclawskiego, PL. GrunWaldzki 2/4, 50-384 Wroclaw, Poland

Dipartimento di Matematica, Politecnico di Torino, Corso Duca degli Abruzzi 24, 10129 TORINO, ITALY

E-mail address: FRICCI@POLITO.IT 